\title{
Special volume on 'Current Trends in Queueing Theory' of the second ECQT conference
}

\author{
Rhonda Righter $^{1}$ • Ina Maria Verloop ${ }^{2,3}$
}

This volume is dedicated to work selected from the 112 presentations at the second, biannual, European Conference on Queueing Theory, held in Toulouse, France, July 18-20, 2016. The nine papers in this special issue have been chosen, revised, and edited for publication through a careful refereeing process. There will be a second, companion volume, with a few more papers, shortly. QUESTA also generously published a special issue of papers selected from the first ECQT meeting (vol. 82, issues 1 and 2, 2016). These special issues would not have been possible without the help of the Technical Program Committee of ECQT and other anonymous referees, and the support of Editorin-Chief Sergey Foss.

The next ECQT meeting will be in Jerusalem, July 24, 2018.

The papers in this volume can be grouped into three general areas. Three of the papers concentrate on extensions to single-server queueing models, three study multiserver and multi-queue models, and three focus on systems with strategic customers.

Abhishek, Boon, Boxma and Núñez Queija study service systems with correlated service times. Special attention is paid to the classical single-server queue with batch arrivals and semi-Markov service times, where the sequence of service times is governed by a modulating process. The authors use generating function techniques to study the transient and stationary queue-length distributions. Numerical evidence shows

$凶$ Rhonda Righter

RRighter@IEOR.Berkeley.edu

Ina Maria Verloop

verloop@irit.fr

1 Department of Industrial Engineering and Operations Research, University of California, Berkeley, CA, USA

2 CNRS, IRIT, Toulouse, France

3 Université de Toulouse, INP, Toulouse, France 
surprising effects, for example, that increasing variability may reduce the mean queue length and that correlated arrivals can lead to large mean queue lengths. Yajima and Phung-Duc also consider a batch arrival queue. The special feature in their model is that the speed of the server is proportional to the number of customers in the system. In addition, the server is turned off immediately if the system becomes empty, and a setup time occurs when a new batch of customers arrives. The authors describe the stability condition and obtain the PGF of the number of customers and the LST of the steady-state sojourn time.

Frostig and Keren-Pinhasik consider a financial risk model that is dual to a queueing model and that describes the amount of capital of a company with fixed expense rate and occasional income inflow. Parisian ruin is studied, where ruin is declared once the process stays continuously below zero for a given period. The authors derive the Laplace transforms of the time to ruin and of the time that the capital is negative. Using the risk-queueing duality relationship, the authors characterize quantities related to the busy period, idle period, and cycle maximum in the G/M/1 queue.

In the paper by Boon, Boxma, Kella, and Miyazawa, the classical result for the steady-state queue-length distribution of a single-class queueing system is extended to the multidimensional setting. In particular, the authors derive distributional relations between the steady-state distribution of the queue length just before an arrival and that just after a departure epoch. These queue-length balance equations allow for simple derivations of known results for polling systems, as well as new results for some priority queueing systems.

The paper of Koops, Boxma and Mandjes considers networks of infinite server queues with shot-noise-driven arrival intensities. The authors derive an explicit expression for the transform of the joint distribution of the number of customers and the shot-noise arrival rate process, and they obtain heavy-traffic asymptotics by using a linear scaling of the shot intensity.

Bonald, Comte, Shah, and de Veciana study a system of multiple processor-sharing queues whose capacity set is a particular type of polytope. The queues have statedependent service rates as given by the balanced fairness allocation. The authors derive polynomial time methods for computation of performance metrics, which facilitate the derivation of stochastic bounds for a large class of systems.

Hassin and Snitkovsky analyze a noncooperative multi-player game in a queueing network, in which customers have no information about queue lengths and server availability. With probability $p$ customers join an infinite-buffer first-come first-served single-server queue. In order to avoid waiting, with probability $1-p$ customers attempt to join a second loss queue that offers service with no delay if the server is idle. This is costly, and, if the server is busy, the customer is blocked and rerouted to the first queue. The authors prove that a unique symmetric Nash equilibrium always exists, and they study the Price of Anarchy. Burnetas, Economou, and Vasiliadis study strategic customer behavior in the presence of delayed queue-length information. Customers are provided with periodic announcements of their current positions in queue at rate $\theta$, at which time they may choose to renege. Upon arrival, customers must decide whether to balk or join. The authors prove the existence and uniqueness of an equilibrium strategy and provide a scheme for social optimization and coordination. In the paper of Kerner, Sherzer, and Yanco, the information is imperfect, rather than delayed. They 
study ticket queues, in which arriving customers obtain a ticket number and see the ticket number of the customer who most recently entered service, but not the number who have balked. Based on this information, customers decide whether or not to join the queue. The authors show that, in contrast to the classical, fully observable queue, a single threshold strategy (balk if and only if the difference in the two ticket numbers is greater than a threshold) cannot be an equilibrium strategy. 